\title{
Optimal Algorithms for the Multiple Query Problem on Reconfigurable Meshes, with Applications
}

\author{
Venkatavasu Bokka, Member, IEEE Computer Society, \\ Koji Nakano, Member, IEEE Computer Society, \\ Stephen Olariu, Member, IEEE Computer Society, \\ James L. Schwing, Member, IEEE Computer Society, and \\ Larry Wilson, Member, IEEE Computer Society
}

\begin{abstract}
The main contribution of this work is to show that a number of fundamental and seemingly unrelated problems in database design, pattern recognition, robotics, computational geometry, and image processing can be solved simply and elegantly by stating them as instances of a unifying algorithmic framework that we call the Multiple Query problem. The Multiple Query problem (MQ, for short) is a 5-tuple $(\mathcal{Q}, \mathcal{A}, \mathcal{D}, \phi, \oplus)$, where $\mathcal{Q}$ is a set of queries, $\mathcal{A}$ is a set of items, $\mathcal{D}$ is a set of solutions, $\phi: \mathcal{Q} \times \mathcal{A} \rightarrow \mathcal{D}$ is a function, and $\oplus$ is a commutative and associative binary operator over $\mathcal{D}$. The input to the MQ problem consists of a sequence $Q=$

$\left\langle q_{1}, q_{2}, \ldots, q_{m}\right\rangle$ of $m$ queries from $\mathcal{Q}$ and of a sequence $A=\left\langle a_{1}, a_{2}, \ldots a_{n}\right\rangle$ of $n$ items from $\mathcal{A}$. The goal is to compute, for every query $q_{i}$ $(1 \leq i \leq m)$ its solution defined as $\phi\left(q_{i}, A\right)=\phi\left(q_{i}, a_{1}\right) \oplus \phi\left(q_{i}, a_{2}\right) \oplus \cdots \oplus \phi\left(q_{i}, a_{n}\right)$. We begin by discussing a generic algorithm that solves a large class of MQ problems in $O(\sqrt{m}+f(n))$ time on a reconfigurable mesh of size $\sqrt{n} \times \sqrt{n}$, where $f(n)$ is the time necessary to compute the expression $d_{1} \oplus d_{2} \oplus \cdots \oplus d_{n}$ with $d_{i} \in \mathcal{D}$ on such a platform. We then go on to show that the MQ framework affords us an optimal algorithm for the multiple point location problem on a reconfigurable mesh of size $\sqrt{n} \times \sqrt{n}$. Given a set $A$ of $n$ points and a set $Q$ of $m(m \leq n)$ points in the plane, our algorithm reports, in $O(\sqrt{m}+\log \log n)$ time, all points of $Q$ that lie inside the convex hull of $A$. Quite surprisingly, our algorithm solves the multiple point location problem without computing the convex hull of $A$ which, in itself, takes $\Omega(\sqrt{n})$ time on a reconfigurable mesh of size $\sqrt{n} \times \sqrt{n}$. Finally, we prove an $\Omega(\sqrt{m}+g(n))$ time lower bound for nontrivial MQ problems, where $g(n)$ is the lower bound for evaluating the expression $d_{1} \oplus d_{2} \oplus \cdots \oplus d_{n}$ with $d_{i} \in \mathcal{D}$, on a reconfigurable mesh of size $\sqrt{n} \times \sqrt{n}$.
\end{abstract}

Index Terms-Query processing, reconfigurable mesh, database design, pattern recognition, image processing, computational geometry, robotics, morphology, parallel algorithms.

\section{INTRODUCTION}

$\mathrm{T}$ HE maturation of computer science has exacerbated the need to consolidate isolated algorithms and techniques into general computational paradigms. The benefits of such an undertaking come in two flavors: First, it becomes clear that problems previously treated in isolation from one another belong to the same class in the sense that they can be solved similarly by formulating them as instances of a more general paradigm; second, once established, the paradigm is likely to become a powerful tool that can be used to solve a host of other related problems.

In a number of seemingly unrelated contexts in database design, pattern recognition, image processing, VLSI design,

- V. Bokka, S. Olariu and L. Wilson are with the Department of Computer Science, Old Dominion University, Norfolk, VA 23529.

E-mail: olariu@cs.odu.edu.

- K. Nakano is with the School of Information Science, Japan Advanced Institute of Science and Technology, Tatsunokuchi, Ishikawa 923-1292, Japan.E-mail:knakano@jaist.ac.jp.

- J.L. Schwing is with the Department of Computer Science, Central Washington University, Ellensburg, WA 98926.

E-mail: jschwing@cwu.edu.

Manuscript received 8 Jan. 2001; accepted 6 Mar. 2001.

For information on obtaining reprints of this article, please send e-mail to: tpds@computer.org, and reference IEEECS Log Number 113435. computer vision, and robot navigation, one is given collections $A$ and $Q$ of objects and the goal is either to identify a collective property of the objects in $A \cup Q$ or to find, for each object in $Q$ a subset of $A$ satisfying a certain predicate and so on. In image understanding, computer vision, and computer graphics, for example, in the presence of a scene populated with a collection $A$ of objects, a crucial problem is to identify the visibility horizon for a set $Q$ of observers [2], [5]. A somewhat different problem is of interest in path planning and collision avoidance problems in robotics [9], [11], where navigational courses for a set $Q$ of mobile robots is sought in the presence of a set $A$ of obstacles. In pattern recognition, the well-known classification problem involves comparing an unknown pattern $Q$ against a template $A$ and deciding whether the similarity measure is larger than a certain application-dependent threshold [4]. In facility-location problems one is typically interested in an optimal placement of a set $Q$ of facilities (schools, hospitals, etc.) among a set $A$ of existing sites in such a way that a number of constraints are satisfied [1], [21], [22]. Yet another similar problem arises in integrated circuit design in VLSI, where one is interested in the addition of a set $Q$ of modules meant to enhance the 


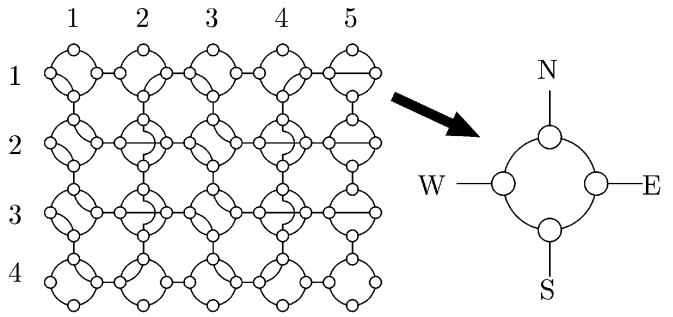

Fig. 1. A reconfigurable mesh of size $4 \times 5$.

functionality of the board $A$. In this latter context, it is customary to state the problem as a visibility problem involving collections $A$ and $Q$ of iso-oriented, nonoverlapping, rectangles in the plane subject to a series of location constraints [20], [21].

In each of these fields, the problems mentioned above are solved, in isolation, using ad hoc techniques. The main contribution of this work is to present a unifying algorithmic framework for solving a number of fundamental and seemingly unrelated problems arising in database design, pattern recognition, image processing, robotics, computer vision, and morphology.

The unifying algorithmic framework is provided by the Multiple Query problem (MQ, for short) that we define next. An MQ problem is a 5-tuple $(\mathcal{Q}, \mathcal{A}, \mathcal{D}, \phi, \oplus)$, where

- $\mathcal{Q}$ is a set of queries,

- $\mathcal{A}$ is a set of items,

- $\mathcal{D}$ is a set of solutions,

- $\phi: \mathcal{Q} \times \mathcal{A} \rightarrow \mathcal{D}$ is a function,

- $\oplus$ is a commutative and associative binary operator over $\mathcal{D}$.

The input to the MQ problem $(\mathcal{Q}, \mathcal{A}, \mathcal{D}, \phi, \oplus)$ is a pair $(Q, A)$, where $Q=\left\langle q_{1}, q_{2}, \ldots, q_{m}\right\rangle$ is a sequence of $m$ queries from $\mathcal{Q}$ and $A=\left\langle a_{1}, a_{2}, \ldots a_{n}\right\rangle$ is a sequence of $n$ items from $\mathcal{A}$. The goal is to compute, for every query $q_{i}(1 \leq i \leq m)$, its solution $\phi\left(q_{i}, A\right)$ defined as:

$$
\phi\left(q_{i}, A\right)=\phi\left(q_{i}, a_{1}\right) \oplus \phi\left(q_{i}, a_{2}\right) \oplus \cdots \oplus \phi\left(q_{i}, a_{n}\right) .
$$

The computational platform that we adopt in this work is the reconfigurable mesh [15]. In essence, a reconfigurable mesh (RM, for short) consists of a mesh-connected multiprocessor machine augmented by the addition of a dynamic bus system whose configuration changes in response to computational and communication needs. More precisely, an RM of size $M \times N$ consists of $M N$ identical processors positioned on a rectangular array with $M$ rows and $N$ columns. Fig. 1 illustrates an RM with $M=4$ rows and $N=5$ columns.

As usual, it is assumed that every processor knows its own coordinates within the mesh. We let $P(i, j)$ denote the processor in row $i$ and column $j$, with $P(1,1)$ in the northwest corner of the mesh. Each processor $P(i, j)$ is connected to its four neighbors $P(i-1, j), P(i+1, j), P(i, j-1)$, and $P(i, j+1)$, provided they exist, and has four ports denoted by N, S, E, and W in Fig. 1. Local connections between these ports can be established, under program control, creating a powerful bus system that changes dynamically to accommodate various computational needs. For practical reasons, only one processor is allowed to broadcast on a given bus at any one time.

In this paper, we assume a reconfigurable mesh model that allows at most two pairs of connections to be set in each processor at any one time. Furthermore, these connections must involve disjoint pairs of ports, as illustrated in Fig. 1. We note that some reconfigurable mesh models proposed in the literature allow processors to fuse any number of their ports [3], [10], [12], [15], [18], [19]. In accord with other workers, we assume ideal bus communications [7], [10], [12], [13], [14]. Although this assumption is inexact, recent experiments with reconfigurable multiprocessor systems [12], [13] seem to indicate that this is a reasonable working hypothesis.

The first main contribution of this paper is to propose a generic algorithm that solves a large class of MQ problems $(\mathcal{Q}, \mathcal{A}, \mathcal{D}, \phi, \oplus)$ involving $m$ queries and $n$ items $(m \leq n)$ on a reconfigurable mesh of size $\sqrt{n} \times \sqrt{n}$. We assume that the sequence $A$ of $n$ items is pretiled, in some order, one item per processor, on a reconfigurable mesh of size $\sqrt{n} \times \sqrt{n}$. Likewise, the sequence $Q$ of $m$ queries from $\mathcal{Q}$ is stored by an arbitrary subset of $m$ processors of the reconfigurable mesh, one query per processor. We shall refer to this situation by saying that the items and queries are in standard position. Our generic algorithm solves a large class of $\mathrm{MQ}$ problems in $O(\sqrt{m}+f(n))$ time on a reconfigurable mesh of size $\sqrt{n} \times \sqrt{n}$, where $f(n)$ if the time needed to compute the expression $d_{1} \oplus d_{2} \oplus \cdots \oplus d_{n}$, with $d_{i} \in \mathcal{D}$, on the same platform. As it turns out, our generic algorithm is optimal whenever the algorithm to evaluate $d_{1} \oplus d_{2} \oplus \cdots \oplus d_{n}$ is. It is also worth noting that most of the operators $\oplus$ of import to applications satisfy either $f(n)=O\left(\log ^{*} n\right)$ or

$$
f(n)=O(\log \log n) .
$$

Our second main contribution is to show that, by using the MQ framework, we can provide simple and elegant solutions to a variety of problems motivated by and finding applications to database design, pattern recognition, robotics, image processing, and computational geometry, among others. The corresponding algorithms run is $O(\sqrt{m}+f(n))$ time, where either $f(n)=O\left(\log ^{*} n\right)$ or $f(n)=O(\log \log n)$, depending on the problem at hand. In particular, we exhibit an optimal algorithm for the multiple point location problem on a reconfigurable mesh of size $\sqrt{n} \times \sqrt{n}$. For a set $A$ of $n$ points in the plane and a set $Q$ of $m$ query points $(m \leq n)$, this algorithm reports all points in $Q$ that lie inside the convex hull of $A$ in $O(\sqrt{m}+\log \log n)$ time. Quite surprisingly, this algorithm solves the multiple point location problem without computing the convex hull of $A$, which, in itself, takes $\Omega(\sqrt{n})$ time on a reconfigurable mesh of size $\sqrt{n} \times \sqrt{n}$.

Our third contribution is to prove an $\Omega(\sqrt{m}+g(n))$ time lower bound for nontrivial MQ problems, where $g(n)$ is the time lower bound for evaluating the expression $d_{1} \oplus d_{2} \oplus \cdots \oplus d_{n}$, for $d_{i} \in \mathcal{D}$, on a reconfigurable mesh of size $\sqrt{n} \times \sqrt{n}$.

The remainder of the paper is organized as follows: In Section 2, we present a generic algorithm for a large class of MQ problems on the RM. Section 3 presents a number of applications of the MQ problem. Section 4 discusses a time 
lower bound for nontrivial MQ problems on the RM. Finally, Section 5 offers concluding remarks and points out directions for further research.

\section{A Generic Algorithm for MQ Problems ON THE RM}

The main goal of this section is to propose a generic algorithm for a comprehensive class of MQ problems that we call root-solvable MQ problems. As it turns out, the vast majority of $\mathrm{MQ}$ problems of practical interest are rootsolvable.

Before presenting the algorithm, however, Section 2.1 discusses basic assumptions about the parameters of the MQ problems that we address in this paper. We also offer a quick refresher of known algorithmic results on reconfigurable meshes that will be instrumental in assessing the complexity of various algorithms in the remainder of this paper.

\subsection{Preliminaries}

In the remainder of this work, unless specified otherwise, we assume that a sequence $A$ of $n$ items is pretiled on an RM of size $\sqrt{n} \times \sqrt{n}$, in arbitrary order, one item per processor. Further, a sequence $Q$ of $m(m \leq n)$ queries is stored by an arbitrary subset of $m$ processors, one query per processor. We make no a priori assumption about the distribution of $Q$. As already mentioned, in Section 1, we shall refer to this arrangement by saying that the items and queries are in standard position.

If the computation of $\phi$ and $\oplus$ is hard, the computing time necessary to evaluate the corresponding expressions will be dominant. Further, if the queries, items, or solutions have so many bits that it takes a lot of time to transfer them, then the data movement time will be dominant.

However, in order to focus on the parallelism inherent in the MQ problems, it is natural to assume that:

- Any element in $\mathcal{Q}, \mathcal{A}$, and $\mathcal{D}$ can be broadcast in $O(1)$ time on a bus,

- For every choice of a query $q \in \mathcal{Q}$ and of an item $a \in \mathcal{A}$, the function $\phi(q, a)$ can be computed in $O(1)$ time by a single processor,

- For every pair $d, d^{\prime} \in D, d \oplus d^{\prime}$ can be computed in $O(1)$ time by a single processor.

First, assume that $m(m \leq n)$ elements are pretiled on a reconfigurable mesh of size $\sqrt{n} \times \sqrt{n}$ in standard position. Under the first assumption above, the following result of Miller et al. [14] allows us to compress the $m$ elements in the northwest submesh of size $\sqrt{m} \times \sqrt{m}$, one element per processor.

Lemma 2.1. Even if $m$ is not known beforehand, an arbitrary set of $m$ elements stored one element per processor in a reconfigurable mesh of size $\sqrt{n} \times \sqrt{n}$, can be moved in $O(\sqrt{m})$ time to the northwest submesh of size $\sqrt{m} \times \sqrt{m}$, one element per processor.

Suppose that $n$ elements $d_{1}, d_{2}, \ldots, d_{n}$ are pretiled on an RM of size $\sqrt{n} \times \sqrt{n}$ in standard position. We say that a, MQ problem is $f(n)$-computable if $d_{1} \oplus d_{2} \oplus \cdots \oplus d_{n}$ can be computed in $f(n)$ time on an RM of size $\sqrt{n} \times \sqrt{n}$. It is easy to see that, for any commutative and associative binary operator $\oplus$, MQ problems are $O(\log n)$-computable on the reconfigurable mesh [14]. However, as the next result shows, for some basic binary operators, the MQ problems are $o(\log n)$-computable.

Lemma 2.2. Consider a reconfigurable mesh of size $\sqrt{n} \times \sqrt{n}$. The following statements hold:

- The task of computing the sum of $n$ binary values is $O\left(\log ^{*} n\right)$-computable [6].

- The task of computing the sum of $n$ b-bit integers is $O\left(\log b+\log ^{*} n\right)$-computable [17].

- The task of finding the minimum or maximum of $n$ reals is $O(\log \log n)$-computable [16].

Suppose that $n$ items $a_{1}, a_{2}, \ldots, a_{n}$ and $n$ queries $q_{1}, q_{2}, \ldots, q_{n}$ are pretiled on an RM of size $\sqrt{n} \times \sqrt{n}$ in standard position. In other words, each processor is storing one item and one query. The corresponding MQ problem can be solved, trivially, in $O(n)$ time as follows: Begin by broadcasting $a_{1}$ to all processors. For every $i(1 \leq i \leq n)$, the processor storing $q_{i}$ computes $\phi\left(q_{i}, a_{1}\right)$. Next, $a_{2}$ is broadcast and the processor storing $q_{i}$ computes $\phi\left(q_{i}, a_{2}\right)$ as well as $\phi\left(q_{i}, a_{1}\right) \oplus \phi\left(q_{i}, a_{2}\right)$. Continuing as above, in $m$ steps, the processor storing $q_{i}$ determines $\phi\left(q_{i}, A\right)$.

Can one do better? Fortunately, the answer is positive for most MQ problems of interest. Sticking to the above setup, we say that the MQ problem is root-solvable if it can be solved in $O(\sqrt{n})$ time. In fact, all the problems discussed in this paper turn out to be root-solvable. For later reference, we note the following lemma, which will be used to show that many MQ problems are root-solvable:

Lemma 2.3. The following tasks can be performed in $O(\sqrt{n})$ time on a regular mesh of size $\sqrt{n} \times \sqrt{n}$ :

- $\quad$ The task of sorting $n$ items [23].

- The task of computing the convex hull of $n$ points in the plane [1].

\subsection{Handling the Case of Root-Solvable MQ Problems}

In this section, we address ourselves to the task of designing a generic algorithm for arbitrary root-solvable MQ problems on reconfigurable meshes.

Suppose that a sequence $Q$ of $m$ queries and a sequence $A$ of $n$ items are pretiled on an RM of size $\sqrt{n} \times \sqrt{n}$ in standard position. Our goal is to show that an arbitrary root-solvable, $f(n)$-computable MQ problem can be solved in $O(\sqrt{m}+f(n))$ time. For this purpose, we begin by assuming that $m$ and $n$ satisfy $m \leq \sqrt{n}$. Later, we will show how to modify the algorithm for $m$ and $n$ satisfying $\sqrt{n}<m \leq n$.

During the course of the algorithm, we perceive the reconfigurable mesh of size $\sqrt{n} \times \sqrt{n}$ as being partitioned into submeshes of various sizes. First, imagine that the reconfigurable mesh is partitioned into $\frac{n}{m^{2}}$ groups $G_{i, j}$ $\left(1 \leq i, j \leq \frac{\sqrt{n}}{m}\right)$, each of size $m \times m$, and refer to Fig. 2 . We refer to the subsequence of items stored by the processors belonging to group $G_{i, j}$ as $A\left(G_{i, j}\right)$. 


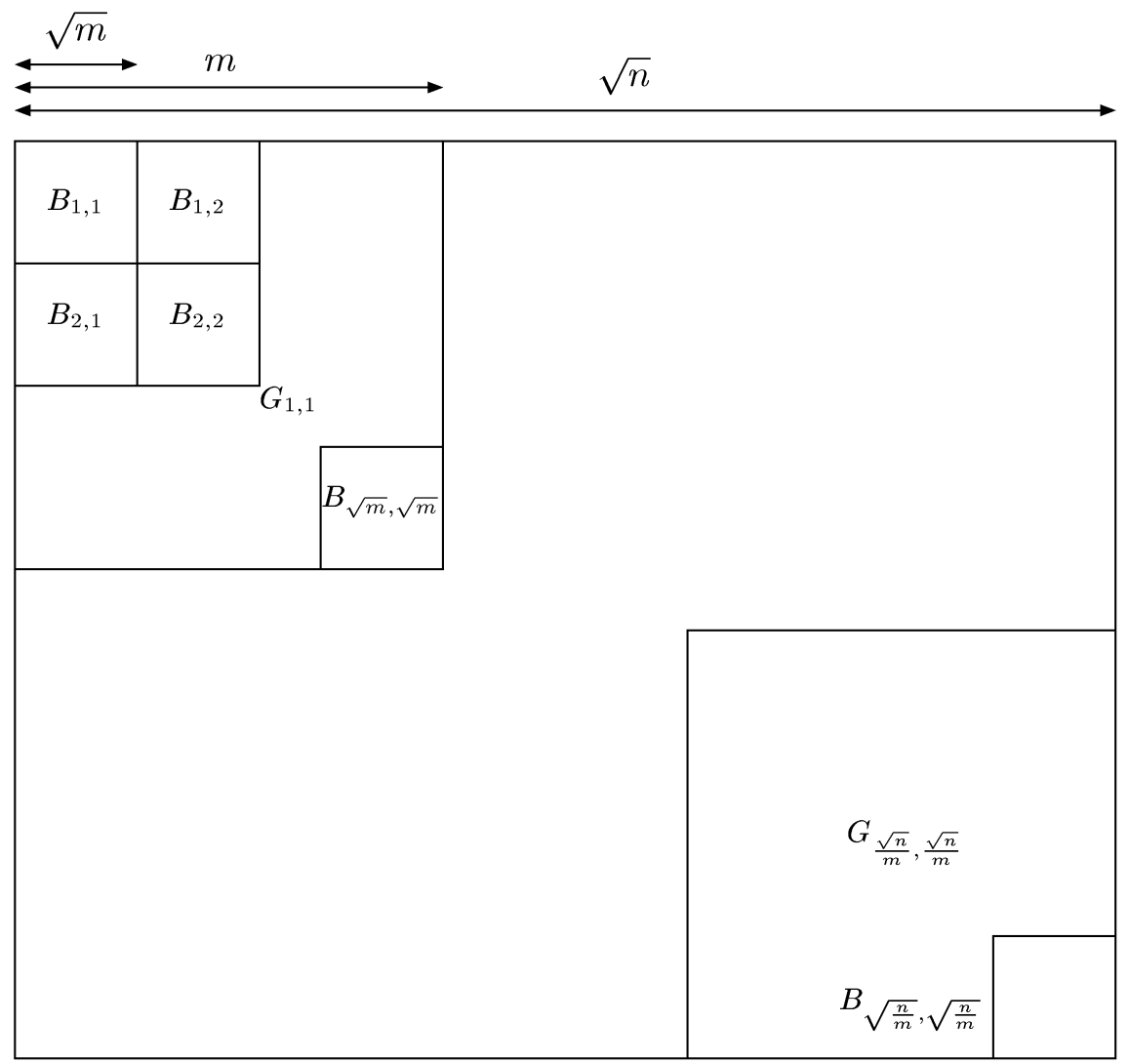

Fig. 2. Illustrating the partition into groups and blocks.

It will also be convenient to view the original reconfigurable mesh as being partitioned into $\frac{n}{m}$ blocks $B_{i, j}$ $\left(1 \leq i, j \leq \sqrt{\frac{n}{m}}\right)$, each of size $\sqrt{m} \times \sqrt{m}$. (It is clear that each group consists of exactly $\sqrt{m} \times \sqrt{m}$ blocks.) As above, we let $A\left(B_{i, j}\right)$ denote the subsequence of items stored by the processors belonging to block $B_{i, j}$.

The following generic algorithm solves any root-solvable, $f(n)$-computable MQ problem with input $(Q, A)$ $(|Q|=m$ and $|A|=n)$ in $O(\sqrt{m}+f(n))$ time.

Step 1. Move the $m$ queries to block $B_{1,1}$ in $O(\sqrt{m})$ time.

Step 2. Replicate the $m$ queries to all the blocks in $O(\sqrt{m})$ time.

Step 3. Solve the MQ problem with input $\left(Q, A\left(B_{i, j}\right)\right)$ $\left(1 \leq i, j \leq \sqrt{\frac{n}{m}}\right)$ local to block $B_{i, j}$ in $O(\sqrt{m})$ time.

Step 4. Combine the solutions obtained in Step 3 to obtain the solution of the MQ problem with input $\left(Q, A\left(G_{i, j}\right)\right)$ $\left(1 \leq i, j \leq \frac{\sqrt{n}}{m}\right)$ in each group $G_{i, j}$ in $O(\sqrt{m})$ time.

Step 5. By combining the solutions obtained in Step 4, solve the original MQ problem with input $(Q, A)$ in $O(\sqrt{m}+$ $\left.f\left(\frac{n}{m^{2}}\right)\right)$ time.

By Lemma 2.1, Step 1 can be performed in $O(\sqrt{m})$ time. Step 2 can be performed very easily as follows: The $m$ queries in block $B_{1,1}$ are broadcast horizontally to blocks $B_{1,2}, B_{1,3}, \ldots, B_{1, \sqrt{\frac{n}{m}}}$. Since $\sqrt{m}$ horizontal buses are available, this task can be performed in $O\left(\frac{m}{\sqrt{m}}\right)=O(\sqrt{m})$ time. Next, the $m$ queries in each of the blocks $B_{1,1}, B_{1,2}, \ldots, B_{1, \sqrt{\frac{n}{m}}}$ are broadcast vertically in $O(\sqrt{m})$ time in the same manner.
Notice that, at the beginning of Step 3, each block $B_{i, j}$ contains the original $m$ queries as well as the $m$ items $A\left(B_{i, j}\right)$. Since block $B_{i, j}$ has $\sqrt{m} \times \sqrt{m}$ processors, the root-solvable MQ problem with input $\left(Q, A\left(B_{i, j}\right)\right)$ can be solved in $O(\sqrt{m})$ time.

To make life easier later, at the end of Step 3, we assign each of the processors of a generic block $B_{i, j}$ to one of the queries. In this capacity, the processor assigned to query $q_{k}(1 \leq k \leq m)$ is responsible for storing $\phi\left(q_{k}, A\left(B_{i, j}\right)\right)$. For reasons that will be clear later, the assignment of processors to queries is as follows: The processors in the rightmost column of $B_{i, j}$ are assigned in top-down order to queries $q_{1}, q_{2}, \ldots, q_{\sqrt{m}}$, the processors in the next column are assigned to queries $q_{\sqrt{m}+1}, q_{\sqrt{m}+2}, \ldots, q_{2 \sqrt{m}}$, and so on. It is clear that by sorting, the processor assigned to query $q_{k}(1 \leq k \leq m)$ receives the value $\phi\left(q_{k}, A\left(B_{i, j}\right)\right)$ in $O(\sqrt{m})$ time [23].

Next, we discuss in detail the resolution of the MQ problem with input $\left(Q, A\left(G_{1,1}\right)\right)$ local to group $G_{1,1}$ in Step 4. The reader will not fail to understand that this is done for convenience only and that the solution of the MQ problems local to the other groups follows the same pattern. Recall that the submesh $G_{1,1}$ consists of $\sqrt{m} \times \sqrt{m}$ blocks $B_{i, j}$ $(1 \leq i, j \leq \sqrt{m})$. The task specific to Step 4 is to compute, for each $q_{k}$,

$$
\begin{aligned}
\phi\left(q_{k}, A\left(G_{1,1}\right)\right)= & \phi\left(q_{k}, A\left(B_{1,1}\right)\right) \oplus \phi\left(q_{k}, A\left(B_{1,2}\right)\right) \\
& \oplus \cdots \oplus \phi\left(q_{k}, A\left(B_{\sqrt{m}, \sqrt{m}}\right)\right) .
\end{aligned}
$$




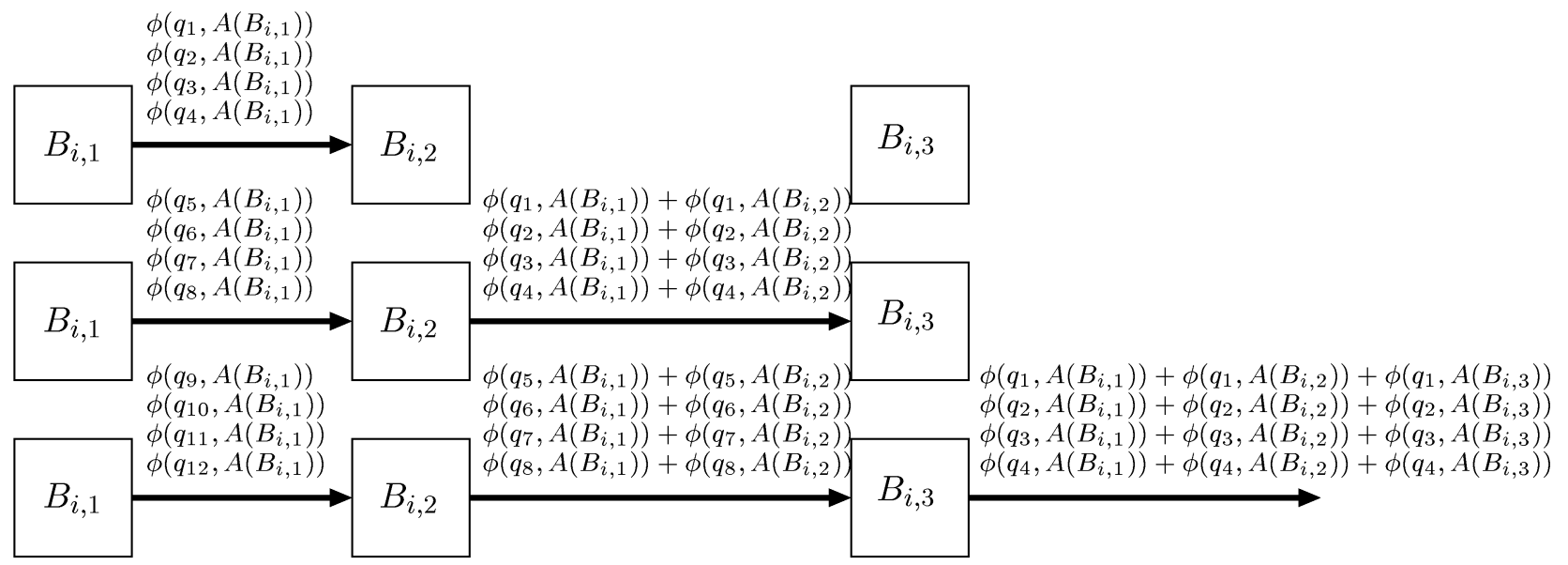

Fig. 3. Illustrating the first three time units of the pipelining in Step 4.

This will be done in two stages. First, in the submesh of size $\sqrt{m} \times m$ consisting of blocks $B_{i, 1}, B_{i, 2}, \ldots, B_{i, \sqrt{m}}$ $(1 \leq i \leq \sqrt{m})$, we compute:

$$
\phi\left(q_{k}, A\left(B_{i, 1}\right)\right) \oplus \phi\left(q_{k}, A\left(B_{i, 2}\right)\right) \oplus \cdots \oplus \phi\left(q_{k}, A\left(B_{i, \sqrt{m}}\right)\right) .
$$

Since adjacent blocks share $\sqrt{m}$ buses, the first stage can be completed in $O(\sqrt{m})$ time by using pipelining, as described next. For an illustration, the reader is referred to Fig. 3.

In the first time unit of the pipelining process,

- the value $\phi\left(q_{k}, A\left(B_{i, 1}\right)\right)(1 \leq k \leq \sqrt{m})$ stored by the processors in the first (i.e., rightmost) column of block $B_{i, 1}$ assigned to query $q_{k}$ is sent to the processor assigned to query $q_{k}$ in the first column of block $B_{i, 2}$,

We note that, upon receiving $\phi\left(q_{k}, A\left(B_{i, 1}\right)\right)$, the processor assigned to query $q_{k}$ in the first column of block $B_{i, 2}$ computes $\phi\left(q_{k}, A\left(B_{i, 1}\right)\right) \oplus \phi\left(q_{k}, A\left(B_{i, 2}\right)\right)$. In the second time unit,

- the value $\phi\left(q_{k}, A\left(B_{i, 1}\right)\right)(\sqrt{m}+1 \leq k \leq 2 \sqrt{m})$ stored by the processors in the second column (in left to right order) of block $B_{i, 1}$ assigned to query $q_{k}$ is sent to the processor assigned to query $q_{k}$ in the second column of block $B_{i, 2}$,

- the value

$$
\phi\left(q_{k}, A\left(B_{i, 1}\right)\right) \oplus \phi\left(q_{k}, A\left(B_{i, 2}\right)\right)
$$

$(1 \leq k \leq \sqrt{m})$ computed by the processor assigned to query $q_{k}$ in the first column of block $B_{i, 2}$ is sent to the processor assigned to query $q_{k}$ in block $B_{i, 3}$.

This pipelining process continues as described until, eventually, the expression in (1) is accumulated at the processor of block $B_{i, \sqrt{m}}$ in charge of query $q_{k}(1 \leq k \leq m)$. Clearly, this first stage terminates in $O(\sqrt{m})$ time.

The second stage repeats the pipelining of the first stage in the vertical strip of size $m \times \sqrt{m}$ involving the blocks $B_{1, \sqrt{m}}, B_{2, \sqrt{m}}, \ldots, B_{\sqrt{m}, \sqrt{m}}$. At the end of the second stage, the value $\phi\left(q_{k}, A\left(G_{1,1}\right)\right)$ will be available at the processor assigned to $q_{k}$ in block $B_{\sqrt{m}, \sqrt{m}}$. Thus, Step 4 can be completed in $O(\sqrt{m})$ time and, for every query $q_{k}$ $(1 \leq k \leq m), \phi\left(q_{k}, A\left(G_{i, j}\right)\right)$ is obtained in $G_{i, j}$.
In preparation for the processing in Step 5, we find it convenient to assign the $m$ processors on the main diagonal of each group $G_{i, j}$ to the $m$ queries $q_{1}, q_{2}, \ldots, q_{m}$. With this assignment out of the way, we arrange for the partial solution $\phi\left(q_{k}, A\left(G_{i, j}\right)\right)$ corresponding to query $q_{k}$ to be stored by the $k$ th diagonal processor, say in left-to-right order. It is clear that the data movement involved in sending the various partial solutions to the diagonal processors can be completed in at most $O(\sqrt{m})$ time.

Finally, the task specific to Step 5 is to compute, for every query $q_{k}$,

$$
\begin{gathered}
\phi\left(q_{k}, A\right)=\phi\left(q_{k}, A\left(G_{1,1}\right)\right) \oplus \phi\left(q_{k}, A\left(G_{1,2}\right)\right) \\
\oplus \cdots \oplus \phi\left(q_{k}, A\left(G_{\frac{\sqrt{n}}{m}, \frac{\sqrt{n}}{m}}\right)\right) .
\end{gathered}
$$

At this time, we perceive the original mesh as a collection of $m$ submeshes each of size $\frac{\sqrt{n}}{m} \times \frac{\sqrt{n}}{m}$, as illustrated in Fig. 4 .

Recall that, by the data movement at the end of Step 4, the diagonal processors in each $G_{i, j}$ store the partial results; the remaining processors in each submesh provide the buses for connecting the diagonal processors. Each of the resulting meshes of size $\frac{\sqrt{n}}{m} \times \frac{\sqrt{n}}{m}$ is dedicated to one of the $m$

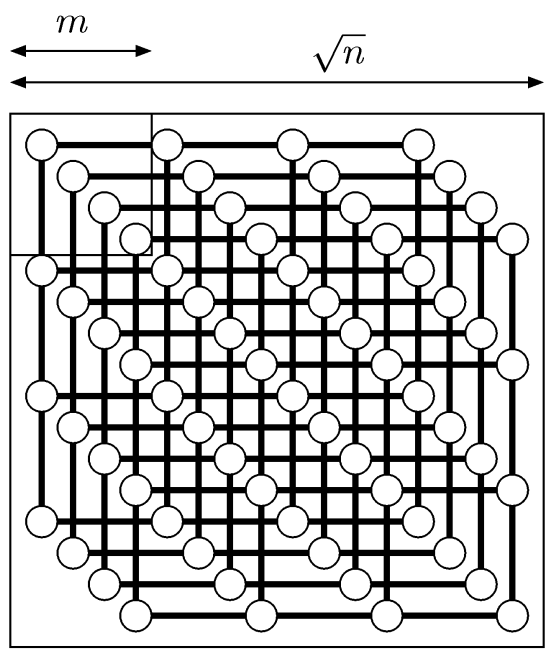

Fig. 4. Illustrating the embedding of $m$ submeshes into the RM. 
queries. In the submesh dedicated to query $q_{k}(1 \leq k \leq m)$, the expression of $\phi\left(q_{k}, A\right)$ in (2) is computed in $O\left(f\left(\frac{n}{m^{2}}\right)\right) \subseteq$ $O(f(n))$ time.

We just proved that an arbitrary root-solvable, $f(n)$-computable MQ problem with input $(Q, A)$ can be solved in $O\left(\sqrt{m}+f\left(\frac{n}{m^{2}}\right)\right)=O(\sqrt{m}+f(n))$ time, provided that $m \leq \sqrt{n}$.

The case $\sqrt{n} \leq m \leq n$ is very similar. The main difference is that we no longer need to view the original reconfigurable mesh partitioned into groups. The only partition is that into blocks. Steps 1, 2, and 3 are the same as above. In Step 4, the first stage of the pipelining process is done is horizontal strips of size $\sqrt{m} \times \sqrt{n}$, with a generic such strip involving the blocks $B_{i, 1}, B_{i, 2}, \ldots, B_{i, \sqrt{n}}$. The pipelining of the first stage terminates in $O\left(\sqrt{\frac{n}{m}}\right) \subseteq O(\sqrt{m})$ time. Likewise, the second stage of the pipelining process involves a vertical strip of size $\sqrt{n} \times \sqrt{m}$. As before, the second stage terminates in $O\left(\sqrt{\frac{n}{m}}\right) \subseteq O(\sqrt{m})$ time. Clearly, the solutions of all the queries are available at the end of Step 4 and, therefore, Step 5 is no longer necessary.

To summarize, we have the following general result:

Theorem 2.4. An arbitrary root-solvable, $f(n)$-computable $M Q$ problem involving $m$ queries and $n$ items, $(m \leq n)$, pretiled, in standard position, on a reconfigurable mesh of size $\sqrt{n} \times \sqrt{n}$ can be solved in $O(\sqrt{m}+f(n))$ time.

\section{Applications}

The main goal of this section is to show that many interesting-and seemingly unrelated-problems in database design, pattern recognition, image processing, VLSI design, computer vision, and robot navigation can be solved simply and elegantly by stating them as nontrivial MQ problems. Importantly, the solution of each of the problems we discuss is obtained by instantiating, in a suitable fashion, the generic algorithm discussed in Section 2.

The general approach that will be taken for each problem is to

- show that it is root-solvable and

- identify the particular function $f(n)$ for which the problem is $f(n)$-computable.

To streamline the notation, in the remainder of this section, we will let $\mathcal{R}$ and $\mathcal{N}$ denote the sets of all real and natural numbers, respectively.

\subsection{Rank Related Problems}

\subsubsection{Multiple Ranking Problem}

Let $Q=\left\langle q_{1}, q_{2}, \ldots, q_{m}\right\rangle$ and $A=\left\langle a_{1}, a_{2}, \ldots, a_{n}\right\rangle$ be sequences of real numbers. The multiple ranking problem asks to compute the rank of each query $q_{i}(1 \leq i \leq m)$ with respect to $A$, that is, the number of items in $A$ strictly smaller than $q_{i}$. The multiple ranking problem corresponds to the MQ problem $(\mathcal{R}, \mathcal{R}, \mathcal{N}, \phi,+)$, where

$$
\phi(q, a)= \begin{cases}1 & \text { if } a<q \\ 0 & \text { otherwise. }\end{cases}
$$

To argue that the multiple ranking problem is root-solvable, consider $n$ queries in $Q$ and $n$ items in $A$ are pretiled on a reconfigurable mesh of size $\sqrt{n} \times \sqrt{n}$ in standard form. Also, without loss of generality, we assume that all real numbers in $Q$ and $A$ are distinct. We must show that every query $q$ in $Q$ can determine in $O(\sqrt{n})$ time its rank $\phi(q, A)$ with respect to $A$. For this purpose we separately sort $Q$ and $Q \cup A$. By Lemma 2.3, these tasks can be completed in $O(\sqrt{n})$ time. From the sorted versions of $Q$ and $Q \cup A$, we can obtain, for every $q \in Q$, the rank $\phi(q, Q)$ of $q$ with respect to $Q$, as well as the $\operatorname{rank} \phi(q, Q \cup A)$ of $q$ with respect $Q \cup A$. Since all the real numbers in $Q$ and $A$ are distinct, it is clear that $\phi(q, A)=\phi(q, Q \cup A)-\phi(q, Q)$, confirming that the multiple ranking problem is root-solvable.

Since, by Lemma 2.2, the sum of $n$ binary values can be computed in $O\left(\log ^{*} n\right)$ time, it follows that the multiple ranking problem is $O\left(\log ^{*} n\right)$-computable. Thus, we have proven the following consequence of Theorem 2.4.

Corollary 3.1. The multiple ranking problem can be solved in $O\left(\sqrt{m}+\log ^{*} n\right)$ time on an RM of size $\sqrt{n} \times \sqrt{n}$.

\subsubsection{Closest Number Problem}

Let $Q=\left\langle q_{1}, q_{2}, \ldots, q_{m}\right\rangle$ and $A=\left\langle a_{1}, a_{2}, \ldots, a_{n}\right\rangle$ be two sequences of real numbers. The closest number problem asks to find, for each query $q_{i}$, the smallest number $a_{j}$ in $A$ satisfying $a_{j}>q_{i}$. The closest number problem corresponds to the MQ problem $(\mathcal{R}, \mathcal{R}, \mathcal{R}, \phi, \min )$, where $\min$ is the "minimum" operator and,

$$
\phi(q, a)=\left\{\begin{array}{cl}
a & \text { if } q<a \\
+\infty & \text { otherwise }
\end{array}\right.
$$

To see that the closest number problem is root-solvable, consider $n$ queries in $Q$ and $n$ items in $A$ are pretiled on a reconfigurable mesh of size $\sqrt{n} \times \sqrt{n}$ in standard form. Again, without loss of generality, we assume that all real numbers in $Q$ and $A$ are distinct. As in the multiple ranking problem, we sort separately $Q, A$, and $Q \cup A$ in $O(\sqrt{n})$ time. From the sorted versions of $Q$ and $Q \cup A$, we can obtain the rank $r(q)$ of $q$ with respect to $A$. Once this is available, we retain the $r(q)$ th item in the sorted version of $A$, which is exactly $\phi(q, A)$. Thus, the closest number problem is root-solvable.

Since, by Lemma 2.2, the maximum of $n$ real numbers can be computed in $O(\log \log n)$ time, the min operator used in the closest number problem is $O(\log \log n)$-computable. Thus, we have proven the following consequence of Theorem 2.4:

Corollary 3.2. The closest number problem can be solved in $O(\sqrt{m}+\log \log n)$ time on an RM of size $\sqrt{n} \times \sqrt{n}$.

\subsection{Image Related Problems}

\subsubsection{Histogramming of Gray-Level Images}

Let $Q=\left\langle q_{1}, q_{2}, \ldots, q_{m}\right\rangle$ be a sequence of $m$ gray levels and let $A=\left\langle a_{1}, a_{2}, \ldots, a_{n}\right\rangle$ be a gray-level image of size $\sqrt{n} \times \sqrt{n}$ pretiled one pixel per processor onto a reconfigurable mesh of the same size. The histogramming problem of an $m$-gray-level image is the MQproblem $(\mathcal{N}, \mathcal{N}, \mathcal{N}, \phi,+)$, where $\phi(q, a)=1$ if the gray-level intensity of pixel $a$ is $q$ and 0 otherwise. It is clear that, once we sort the pixels by their gray levels, we can 
compute the number of pixels of each gray level in constant time. Thus, the histogramming problem is root-solvable. It is also easy to see that the problem is $O\left(\log ^{*} n\right)$-computable. Hence, by Theorem 2.4 and Lemma 2.2, we have the following result:

Corollary 3.2. The task of computing the histogram of a graylevel image of size $\sqrt{n} \times \sqrt{n}$ can be solved in $O\left(\sqrt{m}+\log ^{*} n\right)$ time on an RM of size $\sqrt{n} \times \sqrt{n}$.

Several histogramming algorithms have been presented on the RM. The best previously known known algorithm, which was proposed by Jang et al. [6], runs in $O(\sqrt{m}+$ $\left.\log ^{*} n\right)$ time. Our algorithm matches the time complexity of Jang et al.'s algorithm, while, at the same time, being simpler and easier to understand.

\subsubsection{Range Query Problem}

Let $Q=\left\langle q_{1}, q_{2}, \ldots, q_{m}\right\rangle$ be a sequence of $m$ ranges of real numbers such that $q_{i}=\left[s_{i}, t_{i}\right],\left(s_{i}<t_{i}\right)$, and let

$$
A=\left\langle a_{1}, a_{2}, \ldots, a_{n}\right\rangle
$$

be a sequence of $n$ real numbers. The range query problem asks to find the number of items of $A$ that fall in the range of each $q_{i}$, that is, $\left|\left\{a_{j} \mid s_{i} \leq a_{j} \leq t_{i}\right\}\right|$. Note that the ranges may not be disjoint. The range query problem is a common generalization of both multiple ranking and histogramming. The problem corresponds to the $\mathrm{MQ}$ problem $(\mathcal{R} \times \mathcal{R}, \mathcal{R}, \mathcal{N}, \phi,+)$, where

$$
\phi([s, t], a)= \begin{cases}1 & \text { if } s \leq a \leq t \\ 0 & \text { otherwise }\end{cases}
$$

We observe that, once the rank $\operatorname{rank}\left(s_{i}\right)$ of $s_{i}$ and the rank $\operatorname{rank}\left(t_{i}\right)$ of $t_{i}$ with respect to $A$ are available, we can compute $\operatorname{rank}\left(t_{i}\right)-\operatorname{rank}\left(s_{i}\right)$, which is exactly the solution $\phi\left(\left[s_{i}, t_{i}\right], A\right)$ of query $q_{i}$. Since the multiple ranking problem is root-solvable, the range query problem is also rootsolvable. Moreover, our arguments for the multiple ranking problem carry over to show that the range query problem is $O\left(\log ^{*} n\right)$-computable. Thus, Theorem 2.4 implies the following result:

Corollary 3.5. The range query problem can be solved in $O\left(\sqrt{m}+\log ^{*} n\right)$ time on an RM of size $\sqrt{n} \times \sqrt{n}$.

\subsubsection{Stabbing Problem}

Let $Q=\left\langle q_{1}, q_{2}, \ldots, q_{m}\right\rangle$ be a sequence of $m$ real numbers and let $A=\left\langle a_{1}, a_{2}, \ldots, a_{n}\right\rangle$ be a sequence of $n$ ranges of real numbers such that $a_{j}=\left[s_{j}, t_{j}\right],\left(s_{j}<t_{j}\right)$. The stabbing problem asks to compute, for each query $q_{i}$, the number of ranges in $A$ that include $q_{i}$, that is, $\left|\left\{a_{j} \mid s_{j} \leq q_{i} \leq t_{j}\right\}\right|$. It is clear that the stabbing problem can be stated as the MQ problem $(\mathcal{R}, \mathcal{R} \times \mathcal{R}, \mathcal{N}, \phi,+)$, where

$$
\phi(q,[s, t])= \begin{cases}1 & \text { if } s \leq q \leq t \\ 0 & \text { otherwise }\end{cases}
$$

We note here that the stabbing problem can be stated in a different way as follows: Let $\phi_{1}$ and $\phi_{2}$ be the functions such that
- $\quad \phi_{1}(q,[s, t])=1$ if $s \leq q$ and 0 otherwise and

- $\phi_{2}(q,[s, t])=1$ if $t<q$ and 0 otherwise.

Clearly, $\left(\mathcal{R}, \mathcal{R} \times \mathcal{R}, \mathcal{N}, \phi_{1},+\right)$ and $\left(\mathcal{R}, \mathcal{R} \times \mathcal{R}, \mathcal{N}, \phi_{2},+\right)$ are essentially the multiple ranking problem. Further, it should be clear that $\phi(q, A)=\phi_{1}(q, A)-\phi_{2}(q, A)$ always holds. Hence, by solving the multiple ranking problem twice, one can obtain the solution of the stabbing problem. Thus, we have the following result:

Corollary 3.5. The stabbing problem can be solved in $O(\sqrt{m}+$ $\log ^{*} n$ ) time on an RM of size $\sqrt{n} \times \sqrt{n}$.

\subsection{Geometric Problems}

\subsubsection{Multiple Closest Point Problem}

Let $Q=\left\langle q_{1}, q_{2}, \ldots, q_{m}\right\rangle$ and $A=\left\langle a_{1}, a_{2}, \ldots, a_{n}\right\rangle$ be sequences of points in the plane. The multiple closest point problem asks to find for each query in $Q$ the closest point in $A$. The corresponding MQ problem is

$$
(\mathcal{R} \times \mathcal{R}, \mathcal{R} \times \mathcal{R}, \mathcal{R} \times \mathcal{R} \times \mathcal{R}, \phi, \oplus)
$$

such that

- $\phi(q, a)=\langle d, a\rangle$, where $d$ is the Euclidean distance between $q$ and $a$, and

- $\langle d, a\rangle \oplus\left\langle d^{\prime}, a^{\prime}\right\rangle=\langle d, a\rangle$ if $d \leq d^{\prime}$ and $\left\langle d^{\prime}, a^{\prime}\right\rangle$ otherwise. It is clear that the closest point of $q$ is $a$ if $\langle d, a\rangle=\phi(q, A)$ for some $d$.

Jeong and Lee [8] have shown that the multiple closest point problem can be solved in $O(\sqrt{n})$ time on a regular mesh of size $\sqrt{n} \times \sqrt{n}$. It follows that this problem is rootsolvable. In addition $\oplus$ operator used in this problem is $O(\log \log n)$-computable by Lemma 2.2. Thus, Theorem 2.4 implies the following result:

Corollary 3.6. The multiple closest point problem can be solved in $O(\sqrt{m}+\log \log n)$ time on an RM of size $\sqrt{n} \times \sqrt{n}$.

\subsubsection{Multiple Closest Segment Problem}

Let $Q=\left\langle q_{1}, q_{2}, \ldots, q_{m}\right\rangle$ be a sequence of $m$ points in the plane and let $A=\left\langle a_{1}, a_{2}, \ldots, a_{n}\right\rangle$ be a sequence of $n$ segments. The multiple closest segment problem asks to find for each query point in $Q$ the closest segment in $A$ intersected by a vertical ray emanating from it in the negative $y$-direction. We refer the reader to Fig. 5 for an illustration. Here, the solution to query $q_{2}$ is segment $a_{1}$, the solution to query $q_{3}$ is segment $a_{3}$, while the solution to query $q_{4}$ is $\emptyset$.

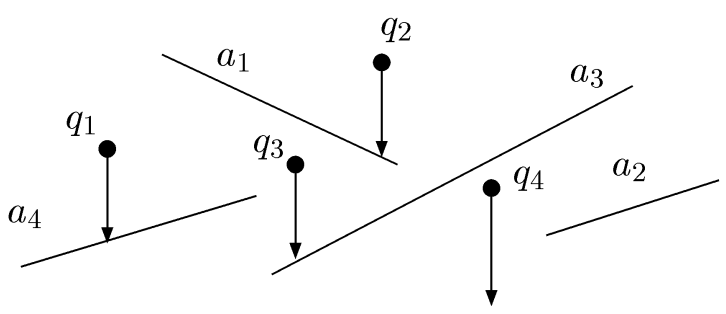

Fig. 5. Illustrating the multiple closest segment problem. 
We observe that the multiple closest segment problem corresponds to the MQ problem

$$
(\mathcal{R} \times \mathcal{R}, \mathcal{R} \times \mathcal{R} \times \mathcal{R} \times \mathcal{R}, \mathcal{R} \times \mathcal{R} \times \mathcal{R}, \phi, \oplus)
$$

such that

- $\phi(q, a)=\langle d, a\rangle$, where $d$ is the vertical distance from point $q$ to segment $a$ if the vertical ray emanating from $q$ intersects $a$ and $+\infty$ otherwise, and,

- $\langle d, a\rangle \oplus\left\langle d^{\prime}, a^{\prime}\right\rangle=\langle d, a\rangle$ if $d \leq d^{\prime}$ and $\left\langle d^{\prime}, a^{\prime}\right\rangle$ otherwise. It should be clear that the closest segment of $q$ is $a$ if $\langle d, a\rangle=$ $\phi(q, A)$ for some $d$.

It is known that the multiple closest segment problem [8] with $n$ points and $n$ segments can be solved in $O(\sqrt{n})$ time on a regular mesh of size $\sqrt{n} \times \sqrt{n}$. It follows that this problem is root-solvable. It can also be argued that the $\oplus$ operator above is $O(\log \log n)$-computable by Lemma 2.2 . Thus, Theorem 2.4 implies the following result:

Corollary 3.7. The multiple closest segment problem involving $m$ points and $n$ segments can be solved in $O(\sqrt{m}+\log \log n)$ time on an RM of size $\sqrt{n} \times \sqrt{n}$.

\subsubsection{Furthest Visible Half-Line Problem}

Let $Q=\left\langle q_{1}, q_{2}, \ldots, q_{m}\right\rangle$ be a sequence of $m$ points in the plane and let $A=\left\langle a_{1}, a_{2}, \ldots, a_{n}\right\rangle$ be a sequence of $n$ top points of vertical half-lines and refer to Fig. 6. The furthest visible half-line problem asks to compute, for each point in $Q$, the visible half-line with, largest $x$-coordinate to its right. For example, the furthest visible half-lines from $q_{1}, q_{2}, q_{3}$, and $q_{4}$ are $a_{4}, a_{3}, a_{1}$, and $a_{5}$, respectively.

Let $\phi\left(q_{i}, a_{j}\right)$ be the angle of the segment $q_{i} a_{j}$ from the downward vertical line from $q_{i}$, as illustrated in Fig. 7. If $\phi\left(q_{i}, A\right)>0$, then the furthest visible half-line from $q_{i}$ is in the direction $\phi\left(q_{i}, A\right)$. If $\phi\left(q_{i}, A\right) \leq 0$, then there exists no half-line in the right hand side of $q_{i}$. It is not hard to see that the furthest visible half-line problem corresponds to the MQ problem $(\mathcal{R} \times \mathcal{R}, \mathcal{R} \times \mathcal{R},[-\pi, \pi), \phi$, max $)$.

As we are going to prove in Lemma 3.10, in the following section, the furthest visible half-line problem is rootsolvable. Further, since the maximum of $n$ real numbers can be computed in $O(\log \log n)$ time on an RM of size $\sqrt{n} \times \sqrt{n}$, the problem is $O(\log \log n)$-computable. Thus, Theorem 2.4 and Lemma 2.2 combined imply the following result:

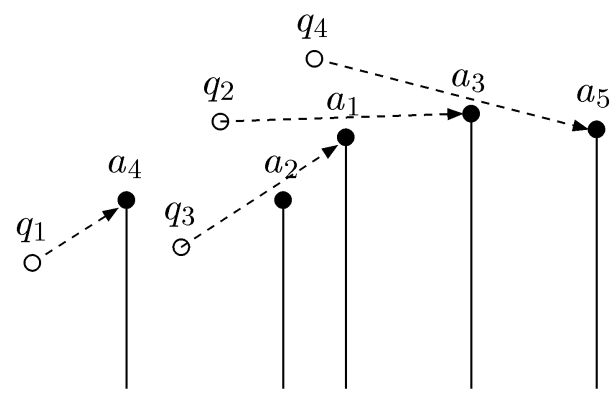

Fig. 6. Illustrating the furthest visible half-line problem.

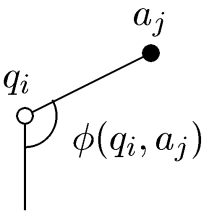

Fig. 7. Illustrating $\phi\left(q_{i}, a_{j}\right)$ for the furthest half-line problem.

Corollary 3.8. The furthest visible half-line problem for $m$ points and $n(\geq m)$ half-lines can be solved in $O(\sqrt{m}+\log \log n)$ time on an RM of size $\sqrt{n} \times \sqrt{n}$.

\subsubsection{Multiple Point Location Problem}

Let $Q=\left\langle q_{1}, q_{2}, \ldots, q_{m}\right\rangle$ and $A=\left\langle a_{1}, a_{2}, \ldots, a_{n}\right\rangle$ be sequences of points in the plane. The multiple point location problem asks to determine, for each point $q \in Q$, whether or not $q$ lies inside the convex hull of $A$. This problem can be solved by using the furthest visible half-line problem as follows: First, solve the furthest visible half-line problem for $Q$ and $A$ and then solve the furthest half-line to its left. Then, for each $q_{i}$, check whether it is below the segment connecting the two furthest visible half-lines. If it is below, then $q_{i}$ is below the upper hull of $A$. Otherwise, it is outside of the convex hull of $A$. Fig. 8 illustrates this. In the same manner, one can check whether $q_{i}$ is above the lower hull. Consequently, the multiple point location problem can be solved by solving the furthest visible half-line problem four times. Thus, we have the following important result:

Corollary 3.9. The multiple point location problem involving $m$ query points and $n(m \leq n)$ data points can be solved in $O(\sqrt{m}+\log \log n)$ time on an RM of size $\sqrt{n} \times \sqrt{n}$.

\subsection{Furthest Visible Half-Line Problem}

This section is devoted to showing that the furthest visible half-line problem is root-solvable. In other words, we are going to show that the furthest visible half-line problems can be solved in $\sqrt{n}$ time for sequences $Q=\left\langle q_{1}, q_{2}, \ldots, q_{n}\right\rangle$ of $n$ points and $A=\left\langle a_{1}, a_{2}, \ldots, a_{n}\right\rangle$ of the tops of $n$ vertical half-lines pretiled on an RM of size $\sqrt{n} \times \sqrt{n}$.

We begin by showing that if $A$ has only $\sqrt{n}$ points, then the furthest visible half-line problems can be solved in $O(\sqrt{n})$ time on an RM of size $\sqrt{n} \times \sqrt{n}$ very easily. First, $a_{1}$ is broadcast to all processors and each processor having $q_{i}$ $(1 \leq i \leq n)$ computes $\phi\left(q_{i}, a_{1}\right)$. Next, $a_{2}$ is broadcast and each processor having $q_{i}$ computes $\phi\left(q_{i}, a_{2}\right)$ and then computes $\phi\left(q_{i}, a_{1}\right) \oplus \phi\left(q_{i}, a_{2}\right)$. Continuing similarly, each processor having $q_{i}$ obtains $\phi\left(q_{i}, A\right)$ in $O(\sqrt{n})$ time provided that $A$ has $\sqrt{n}$ points.

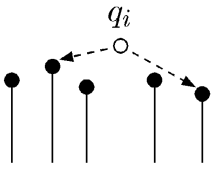

$q_{i}$ is above the upper hull

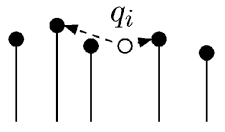

$q_{i}$ is below the upper hull
Fig. 8. Determining whether a point is above or below the upper hull. 


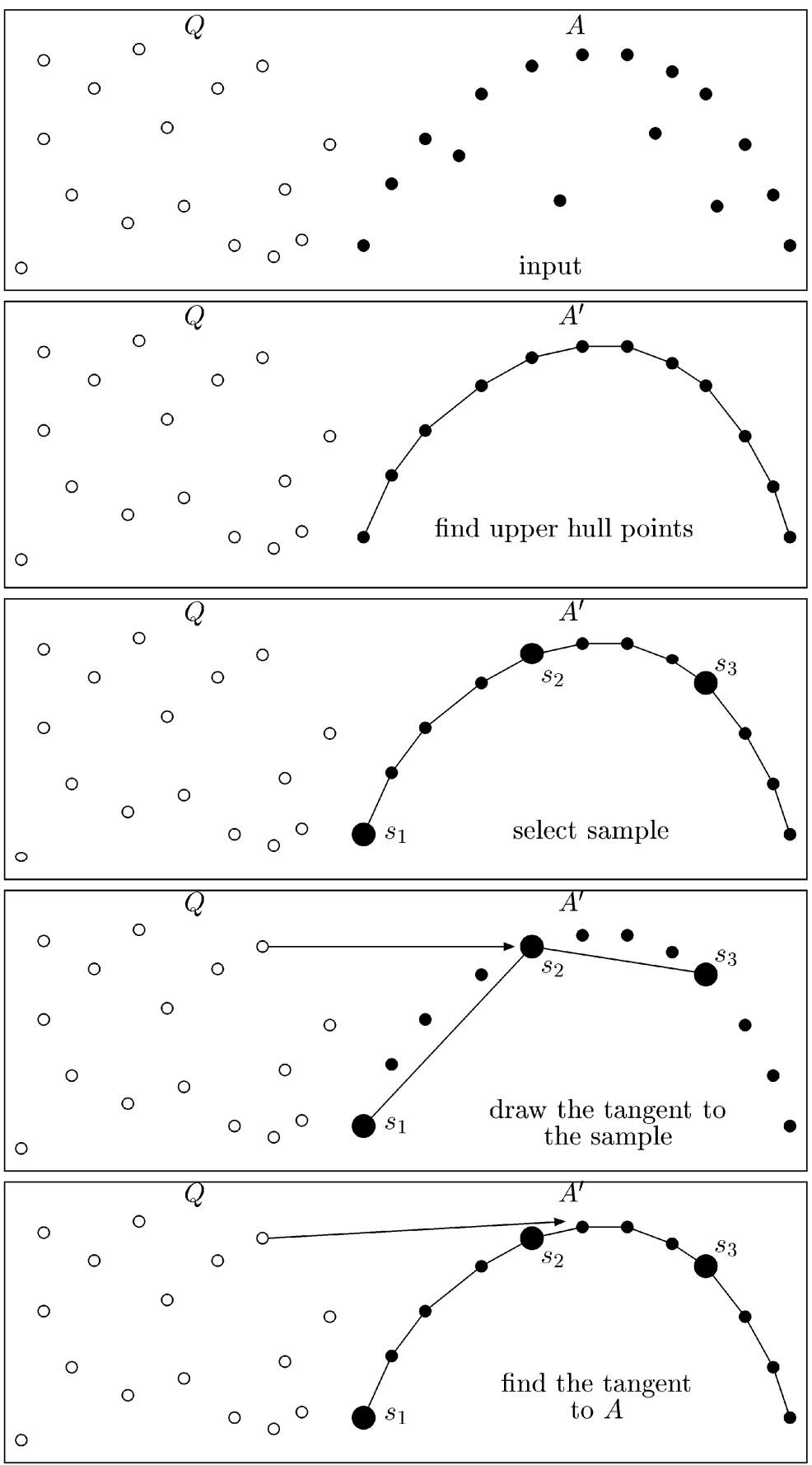

Fig. 9. Illustrating the algorithm for solving the furthest visible half-line problem for separated $Q$ and $A$.

Next, we will show, if $Q$ and $A$ are separated, that is, if all points in $Q$ are to the left of all points in $A$, then the furthest visible half-line problem can be solved in $O(\sqrt{n})$ time, even if both $A$ and $Q$ have $n$ points each. We refer the reader to Fig. 9 for an illustration. First, find the upper hull points in $A$ in $O(\sqrt{n})$ time and remove all nonupper hull points. Then, sort all upper hull points by $x$-coordinate. These tasks can be performed in $O(\sqrt{n})$ time by Lemma 2.3. Let $A^{\prime}=$ $\left\langle a_{1}^{\prime}, a_{2}^{\prime}, \ldots, a_{n^{\prime}}^{\prime}\right\rangle\left(n^{\prime} \leq n\right)$ be the sorted upper hull points thus obtained. Clearly, it is sufficient to find the tangent $q_{i} a_{j}^{\prime}$ from each $q_{i}$ to $A^{\prime}$ to solve the furthest visible half-line problem. For this purpose, partition $A^{\prime}$ into $\frac{n^{\prime}}{\sqrt{n}}$ subsequence $A_{1}^{\prime}, A_{2}^{\prime}, \ldots, A_{\frac{n^{\prime}}{\sqrt{n}}}^{\prime}$ such that each $A_{j}^{\prime}$ has $\sqrt{n}$ points which are consecutive in their $x$-coordinates. Further, let $s_{j}$ $\left(1 \leq j \leq \frac{n^{\prime}}{\sqrt{n}}\right)$ be the leftmost point in $A_{j}^{\prime}$. Next, find the tangent from each point $q_{i}(1 \leq i \leq n)$ in $Q$ to the upper hull $S=\left\langle s_{1}, s_{2}, \ldots, s_{\frac{n^{\prime}}{n}}\right\rangle$. This can be performed in $O\left(\frac{n^{\prime}}{\sqrt{n}}\right) \leq$ $O(\sqrt{n})$ time. We now have the important fact: If $q_{i} s_{j}$ is the tangent from $q_{i}$ to $S$, then the tangent $q_{i} a_{j^{\prime}}$ from $q_{i}$ to $A$ satisfies $a_{j^{\prime}} \in A_{j-1}^{\prime} \cup A_{j^{\prime}}^{\prime}$. In other words, it is sufficient to compute the tangent from $q_{i}$ to $A_{j-1}^{\prime} \cup A_{j}^{\prime}$ to obtain the tangent from $q_{i}$ to $A$. Now, consider that a point $q_{i}$ $(1 \leq i \leq n)$ has a key $j$ if $q_{i} s_{j}$ is the tangent from $q_{i}$ to $S$. Let $Q_{j}\left(1 \leq j \leq \frac{n^{\prime}}{\sqrt{n}}\right)$ be the set of points in $Q$ with key $j$. We assign $\left\lceil\frac{\left.\mid Q_{j}\right]}{\sqrt{n}}\right\rceil$ columns to $Q_{j}$. The total number of columns that are necessary is 

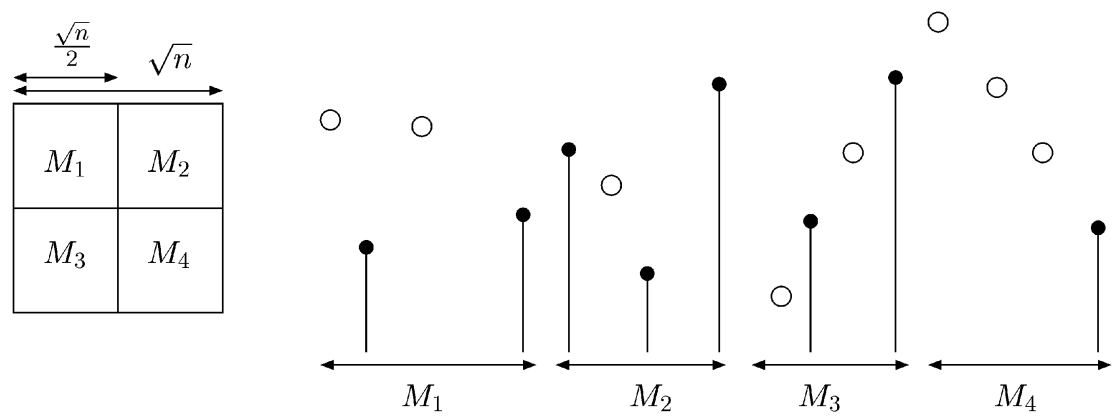

Fig. 10. Partitioning points and lines into four subsets.

$$
\begin{aligned}
\sum_{j=1}^{\frac{n^{\prime}}{\sqrt{n}}}\left\lceil\frac{\left|Q_{j}\right|}{\sqrt{n}}\right] & <\sum_{j=1}^{\frac{n^{\prime}}{\sqrt{n}}}\left(\frac{\left|Q_{j}\right|}{\sqrt{n}}+1\right) \\
& =\frac{\sum_{j=1}^{\frac{n^{\prime}}{\sqrt{n}}}\left|Q_{j}\right|}{\sqrt{n}}+\frac{n^{\prime}}{\sqrt{n}} \\
& \leq 2 \sqrt{n} .
\end{aligned}
$$

Since the RM has $\sqrt{n}$ columns, we use each physical column as two imaginary columns. In other words, each physical column works as two columns by obvious time sharing. Then, this assignment is feasible. By sorting $Q$ for the keys appropriately, we can move points in each $Q_{j}$ to consecutive $\left\lceil\frac{\left|Q_{j}\right|}{\sqrt{n}}\right\rceil$ (imaginary) columns. This can be performed in $O(\sqrt{n})$ time from Lemma 2.3. After that, for each $j\left(1 \leq j \leq \frac{n^{\prime}}{\sqrt{n}}\right)$, broadcast points in $A_{j-1}^{\prime} \cup A_{j}^{\prime}$ in turn to processors having points in $Q$ with key $j$. More precisely, for each $j\left(1 \leq j \leq \frac{n^{\prime}}{\sqrt{n}}\right)$, the $\sqrt{n}$ points in $A_{j}^{\prime}$ broadcast horizontally to every column. The columns having points in $Q_{j}$ and $Q_{j+1}$ receive them. This can be done in $O\left(\frac{n^{\prime}}{\sqrt{n}}\right) \leq$ $O(\sqrt{n})$ time. After that, within the columns having points in $Q_{j}$ broadcast points $A_{j-1}^{\prime} \cup A_{j}^{\prime}$ in turn. Since $\left|A_{j-1}^{\prime} \cup A_{j}^{\prime}\right|=2 \sqrt{n}$, this takes $O(\sqrt{n})$ time. Because each processor storing a point in $Q_{j}$ receives all points $A_{j-1}^{\prime} \cup A_{j}^{\prime}$, it can find the tangent from the point to $A$ in the obvious way. Therefore, if $Q$ and $A$ are separated, the furthest visible half-line problem can be solved in $O(\sqrt{n})$ time on the $\sqrt{n} \times \sqrt{n}$ RM.

Finally, we will show that the visible half-line problem can be solved in $O(\sqrt{n})$ time, even if $Q$ and $A$ are not separated. Partition the $\sqrt{n} \times \sqrt{n}$ RM into four submeshes $M_{1}, M_{2}, M_{3}$, and $M_{4}$ of size $\frac{\sqrt{n}}{2} \times \frac{\sqrt{n}}{2}$ each. By sorting the points $Q \cup A$ for $x$-coordinate, move the leftmost (i.e larger $x$-coordinate) $\frac{n}{2}$ points to $M_{1}$ and the next $\frac{n}{2}$ points to $M_{2}$ and so on, as illustrated in Fig. 10. In each submesh, solve the furthest half-line problem recursively. After that, we execute the following three steps:

1. For each point on $M_{1}$, find the furthest visible halfline on $M_{2}, M_{3}, M_{4}$,

2. For each point on $M_{2}$, find the furthest visible halfline on $M_{3}, M_{4}$, and

3. For each point on $M_{3}$, find the furthest visible half-line on $M_{4}$.
Since the points and half-lines are separated, each of the three steps can be done in $O(\sqrt{n})$ time. By comparing the local solutions obtained by recursive computation and the solution of these three steps in the obvious way, the solution of the furthest visible half-line can be obtained. Let $T(\sqrt{n})$ denote the computing time necessary to solve the problem on the $\sqrt{n} \times \sqrt{n}$ RM. We have the equations $T(\sqrt{n})=$ $T\left(\frac{\sqrt{n}}{2}\right)+O(\sqrt{n})$ and $T(1)=O(1)$. Consequently, $T(\sqrt{n})=$ $O(\sqrt{n})$ and, thus, we have

Lemma 3.10. The furthest visible half-line problem is rootsolvable.

\section{A Time Lower BOUNd FOR NONTRIVIAL MQ PROBLEMS}

This section is devoted to discussing time lower bound results for MQ problems on reconfigurable meshes.

We begin by showing that the closest number problem has an $\Omega(\sqrt{m})$ time lower bound. Recall that the closest number problem with input $(Q, A)$ asks to find, for each real number $q_{i}$ in $Q$, the smallest real number $a_{j}$ in $A$ satisfying $a_{j}>q_{i}$.

Let $A=\left\langle a_{1}, a_{2}, \ldots, a_{n}\right\rangle$ be a sequence of real numbers such that

- for $1 \leq i \leq m, i<a_{i}<i+1$, and $i<a_{m+i}<i+1$ and,

- for $2 m<i \leq n, a_{i}=+\infty$.

Further, let the sequence of $m$ queries be $Q=\langle 1,2, \ldots, m\rangle$. Clearly, for every $i(1 \leq i \leq m)$, we have

$$
\phi(i, A)=\min \left\{a_{i}, a_{m+i}\right\} .
$$

In other words, to get the solution $\phi(i, A)$, we need to compute the minimum of $a_{i}$ and $a_{m+i}$. It follows that, for every $i(1 \leq i \leq m)$, at least one of the processors on the RM has to learn both $a_{i}$ and $a_{m+i}$.

Without loss of generality, we assume that the sequences $a_{1}, a_{2}, \ldots, a_{m}$ and $a_{m+1}, a_{m+2}, \ldots, a_{2 m}$ are stored, one item per processor, in disjoint submeshes $A$ and $B$ both of size $\sqrt{m} \times \sqrt{m}$.

Now, for every $i(1 \leq i \leq m)$, either $a_{i}$ must be sent out of the submesh $A$ or $a_{m+i}$ must be sent out of the submesh $B$. Thus, at least one of the submeshes $A$ or $B$ contains $\frac{m}{2}$ or more items that have to make their way out of the submesh.

Since each of these submeshes is of size $\sqrt{m} \times \sqrt{m}$, at most $4 \sqrt{m}$ items can exit per time unit. It follows that $\frac{\frac{m}{2}}{4 \sqrt{m}}=$ 
$\Omega(\sqrt{m})$ time is necessary for this task. Thus, $\Omega(\sqrt{m})$ is a time lower bound for solving the closest number problem on a reconfigurable mesh of size $\sqrt{n} \times \sqrt{n}$.

Unfortunately, the above lower bound argument does not apply to all MQ problems. To see this, let us define the product-sum problem as follows: As before, $Q$ and $A$ are real numbers. However, we define the function $\phi$ such that $\phi(q, a)=q a$. In this context, the solution of every query $q_{i}$ is $\phi\left(q_{i}, A\right)=q_{i} a_{1}+q_{i} a_{2}+\cdots+q_{i} a_{n}$. Since, on the other hand, $\phi\left(q_{i}, A\right)=q_{i}\left(a_{1}+a_{2}+\cdots+a_{n}\right)$, it follows that we can solve this problem by precomputing the sum $a_{1}+a_{2}+\cdots+a_{n}$ on the RM. If each item has $b$ bits, Lemma 2.2 guarantees that this can be done in $O\left(\log b+\log ^{*} n\right)$ time. Once computed, the sum $a_{1}+a_{2}+\cdots+a_{n}$ is broadcast to all processors and the processor storing $q_{i}$ computes the solution $\phi\left(q_{i}, A\right)$ in $O(1)$ time. Thus, this problem can be solved in $O(\log b+$ $\left.\log ^{*} n\right)$ time which, in general, is much faster than $\Omega(\sqrt{m})$. What happened here, of course, is that the relevant information about the $n$ items $a_{1}, a_{2}, \cdots, a_{n}$ could be "encoded" into one integer $a_{1}+a_{2}+\cdots+a_{n}$ and this integer carries enough information to yield the solution to every query immediately.

The key point of the time lower bound argument for the closest number problem is that at least one processor has to learn both $a_{i}$ and $a_{m+i}$. With this observation in mind, we are now in a position to identify a large class of MQ problems that have an $\Omega(\sqrt{m})$ time lower bound.

We say that $A=\left\langle a_{1}, a_{2}, \ldots, a_{n}\right\rangle$ is a mixed sequence of $B=$ $\left\langle b_{1}, b_{2}, \ldots, b_{n}\right\rangle$ and $B^{\prime}=\left\langle b_{1}^{\prime}, b_{2}^{\prime}, \ldots, b_{n}^{\prime}\right\rangle$ if, for every $i$ $(1 \leq i \leq n), a_{i}=b_{i}$ or $a_{i}=b_{i}^{\prime}$. The MQ problem is said to be nontrivial if there exists a sequence $Q=\left\langle q_{1}, q_{2}, \ldots, q_{m}\right\rangle$ of queries and two sequences $B=\left\langle b_{1}, b_{2}, \ldots, b_{n}\right\rangle$ and $B=$ $\left\langle b_{1}^{\prime}, b_{2}^{\prime}, \ldots, b_{n}^{\prime}\right\rangle$ of $n$ items such that the following two conditions are satisfied:

1. For any two mixed sequences $A=\left\langle a_{1}, a_{2}, \ldots, a_{n}\right\rangle$ a n d $A^{\prime}=\left\langle a_{1}^{\prime}, a_{2}^{\prime}, \ldots, a_{n}^{\prime}\right\rangle$, of $B$ a n d $B^{\prime}$, $\phi\left(q_{i}, A\right)=\phi\left(q_{i}, A^{\prime}\right),(1 \leq i \leq m)$, if and only if, $a_{i}=$ $a_{i}^{\prime}$ and $a_{m+i}=a_{m+i}^{\prime}$

2. For every $i(1 \leq i \leq m)$,

$$
\begin{array}{r}
\phi\left(q_{i}, b_{i}\right) \oplus \phi\left(q_{i}, b_{m+i}\right) \neq \phi\left(q_{i}, b_{i}\right) \oplus \phi\left(q_{i}, b_{m+i}^{\prime}\right) \\
\text { and } \phi\left(q_{i}, b_{i}\right) \oplus \phi\left(q_{i}, b_{m+i}\right) \neq \phi\left(q_{i}, b_{i}^{\prime}\right) \oplus \phi\left(q_{i}, b_{m+i}\right) .
\end{array}
$$

The first condition implies that the solution of query $q_{i}$ depends only on the values $a_{i}$ and $a_{m+i}$ if the input is restricted to mixed sequences of $B$ and $B^{\prime}$. The second condition requires that the solution of query $q_{i}$ changes according to the values of $a_{i}$ and $a_{m+i}$. Hence, at least one processor has to know both $a_{i}$ (or $\phi\left(q_{i}, a_{i}\right)$ ) and $a_{m+i}$ (or $\left.\phi\left(q_{i}, a_{m+i}\right)\right)$.

Using the same argument as for the closest number problem, it is easy to show that every nontrivial MQ problem has an $\Omega(\sqrt{m})$ time lower bound. Consequently, we have proven the following result:

Lemma 4.1. The task of solving a nontrivial $M Q$ problem involving $n$ items and $m$ queries requires $\Omega(\sqrt{m})$ time on a reconfigurable mesh of size $\sqrt{n} \times \sqrt{n}$.

To complete our lower bound analysis, we need to express the complexity of the MQ problem in terms of the hardness of the operator $\oplus$. A binary operator $\oplus$ is said to be $g(n)$-hard if $d_{1} \oplus d_{2} \oplus \cdots \oplus d_{n}$ has an $\Omega(g(n))$ time lower bound on a reconfigurable mesh of size $\sqrt{n} \times \sqrt{n}$. We take note of the following important result proved in [16].

Lemma 4.2. The operators minimum and maximum over the reals are $\Omega(\log \log n)$-hard

The motivation for this stems from the observation that if $Q$ contains a single query $q$, then the task of solving the corresponding MQ problem reduces to the task of computing the expression $\phi\left(q, a_{1}\right) \oplus \phi\left(q, a_{2}\right) \oplus \cdots \oplus \phi\left(q, a_{n}\right)$. Thus, if the operator $\oplus$ is $g(n)$-hard, the above expression requires $\Omega(g(n))$ time on an RM of size $\sqrt{n} \times \sqrt{n}$. Thus, we have the following result:

Theorem 4.3. Any nontrivial, $g(n)$-hard $M Q$ problem involving $m$ queries and $n$ items requires $\Omega(\sqrt{m}+g(n))$ time on a reconfigurable mesh of size $\sqrt{n} \times \sqrt{n}$.

\section{Conclusions and Open Problems}

The main contribution of this work was to show that a number of fundamental-and seemingly unrelated-problems from database design, pattern recognition, robotics, computational geometry, and image processing can be solved simply and elegantly by stating them as instances of a unifying algorithmic framework that we call the Multiple Query (MQ) problem.

We begin by exhibiting a generic algorithm solving a large class of $\mathrm{MQ}$ problems-the so-called, root-solvable problems-involving $m$ queries and $n$ items in $O(\sqrt{m}+$ $f(n)$ ) time on a reconfigurable mesh of size $\sqrt{n} \times \sqrt{n}$, where $f(n)$ is the time necessary to compute $d_{1} \oplus d_{2} \oplus \cdots \oplus$ $d_{n}$ for $d_{i} \in \mathcal{D}$ on such a platform.

We then went on to show that several important problems can be solved optimally and almost effortlessly by stating them as MQ problems. In particular, we proposed an optimal algorithm for the multiple point location problem on a reconfigurable mesh of size $\sqrt{n} \times \sqrt{n}$. For a set $A$ of $n$ points and a set $Q$ of $m$ points in the plane $(m \leq n)$, our algorithm reports, in $O(\sqrt{m}+$ $\log \log n$ ) time, all points of $Q$ that lie inside the convex hull of $A$. Importantly, our algorithm solves the multiple point location problem without computing the convex hull of $A$ which, in itself, takes $\Omega(\sqrt{n})$ time on a reconfigurable mesh of size $\sqrt{n} \times \sqrt{n}$.

Finally, we established an important time lower bound result for a large class of MQ problems-the so-called nontrivial MQ problems. Specifically, we showed that any nontrivial MQ problem involving $m$ queries and $n$ items must take $\Omega(\sqrt{m}+g(n))$ time on a reconfigurable mesh of size $\sqrt{n} \times \sqrt{n}$, where $\Omega(g(n))$ is the time lower bound for evaluating the expression $d_{1} \oplus d_{2} \oplus \cdots \oplus d_{n}$ for $d_{i} \in \mathcal{D}$ on such a platform.

Numerous problems are still open. First, it would be interesting to extend the lower bound result to other classes of MQ problems. Second, we expect that many more problems can find a unifying solution by using our MQ paradigm. This promises to be an exciting area for further investigations. 


\section{ACKNOWLEDGMENTS}

The authors are grateful to four anonymous referees for their extremely thorough reading of the manuscript and for the numerous comments and suggestions that lead to a much better overall quality of the final paper. This work was supported in part by US National Science Foundation grant CCR-9522098 and by US Office of Naval Research grant N00014-97-1-0526.

\section{REFERENCES}

[1] S.G. Akl and K.A. Lyons, Parallel Computational Geometry. Englewood Cliffs, N. J.: Prentice Hall, 1993.

[2] D.H. Ballard and C.M. Brown, Computer Vision Englewood Cliffs, N.. J: Prentice Hall, 1982.

[3] Y.-C. Chen and W.-T. Cheng, "Reconfigurable Mesh Algorithms for Summing up Binary Values and Its Applications," Proc. Fourth Symp. Frontiers of Massively Parallel Computation, pp. 427-433, 1992.

[4] R.O. Duda and P.E. Hart, Pattern Classification and Scene Analysis. New York: Wiley and Sons, 1973.

[5] L.D. Foley, A. van Dam, S.K. Feiner, and J.F. Hughes, Computer Graphics, Principles and Practice, second ed., Reading, Mass.: Addison-Wesley, 1990.

[6] J.-W. Jang, H. Park, and V.K. Prasanna, "A Fast Algorithm for Computing a Histogram on Reconfigurable Mesh," IEEE Trans. Pattern Analysis and Machine Intelligence, vol. 17, pp. 97-106, 1995.

[7] J.-F. Jenq and S. Sahni, "Histogramming on a Reconfigurable Mesh Computer," Proc. Sixth Int'l Parallel Processing Symp., pp. 425-432, 1992.

[8] C.S. Jeong and D.T. Lee, "Parallel Geometric Algorithms on a Mesh-Connected Computer," Algorithmica, vol. 5, pp. 155-177, 1990.

[9] J.-P. Laumond, "Obstacle Growing in a Nonpolygonal World," Information Processing Letters, vol. 25, pp. 41-50, 1987.

[10] H. Li and M. Maresca, "Polymorphic-Torus Network," IEEE Trans. Computers, vol. 38, pp. 1345-1351, 1989

[11] T. Lozano-Perez, "Spatial Planning: A Configurational Space Approach," IEEE Trans. Computers, vol. 32, pp. 108-119, 1983.

[12] M. Maresca, "Polymorphic Processor Arrays," IEEE Trans. Parallel and Distributed Systems, vol. 4, pp. 490-506, 1993.

[13] M. Maresca, H. Li, and P. Baglietto, "Hardware Support for Fast Reconfigurability in Processor Arrays," Proc. Int'l Conf. Parallel Processing, vol. I, pp. 282-289, 1993.

[14] R. Miller, V.K. Prasanna, D. Reisis, and Q.F. Stout, "Parallel Computations on Reconfigurable Meshes," IEEE Trans. Computers, vol. 42, pp. 678-692, 1993.

[15] K. Nakano, "A Bibliography of Published Papers on Dynamically Reconfigurable Architectures," Parallel Processing Letters, vol. 5, pp. 111-124, 1995.

[16] K. Nakano and S. Olariu, "An Efficient Algorithm for Row Minima Computations on Basic Recofigurable Meshes," IEEE Trans. Parallel and Distributed Systems, vol. 9, pp. 561-569, 1998

[17] K. Nakano and K. Wada, "Integer Summing Algorithms on Reconfigurable Meshes," Theoretical Computer Science, vol. 197, pp. 57-77, 1998.

[18] S. Olariu, J.L. Schwing, and J. Zhang, "Optimal Convex Hull Algorithms on Enhanced Meshes," BIT vol. 33, pp. 396-410, 1993

[19] S. Olariu, J.L. Schwing, and J. Ahang, "Fast Computer Vision Algorithms for Reconfigurable Meshes," Proc. Sixth Int'l Parallel Processing Symp., pp. 258-261, 1992.

[20] B.T. Preas and M.J.Lorenzetti eds. Physical Design Automation of VLSI Systems, Menlo Park: Benjamin/Cummings, 1988.

[21] F.P. Preparata and M.I. Shamos, Computational Geometry, An Introduction, Berlin, New York: Springer-Verlag, 1988.

[22] J. Serra, Image Analysis and Mathematical Morphology. New York: Academic Press, 1982.

[23] C.D. Thompson and H.T. Kung, "Sorting on a Mesh-Connected Parallel Computer," Comm. ACM, vol. 20, pp. 263-271, 1977.

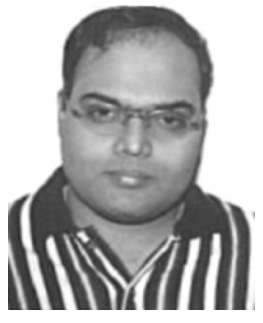

Venkatavasu Bokka received the BTech degree in computer science and engineering in 1992 from the Indian Institute of Technology, Delhi. He received the $\mathrm{PhD}$ degree in computer science in 1996, from Old Dominion University, Norfolk, Virginia. He is currently working at ObjectData, Inc. His research interests include parallel algorithms, computational geometry, and image processing. $\mathrm{He}$ is a member of the IEEE Computer Society.

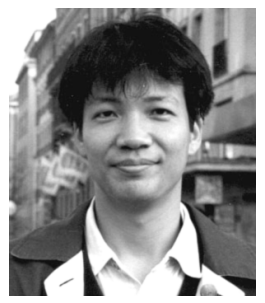

Koji Nakano received the $\mathrm{BE}, \mathrm{ME}$, and $\mathrm{PhD}$ degrees from Osaka University, Japan, in 1987, 1989, and 1992, respectively. From 1992 through 1995, he was a research scientist at the Advanced Research Laboratory, Hitachi Ltd. He worked in the Department of Electrical and Computer Engineering, Nagoya Institute of Technology until 2001. He is currently an associate professor in the School of Information Science, Japan Advanced Institute of Science and Technology. He has published papers in the IEEE Transactions on Parallel and Distributed Systems, Journal of Parallel and Distributed Computing, Theoretical Computer Science, Theory of Computing Systems, Parallel Algorithms and Applications, and IEICE Transactions. $\mathrm{He}$ has served as an editor of Journal of the Information Processing Society of Japan and IEICE Transactions on Information and Systems. $\mathrm{He}$ has served as the program chair or as a committee member of conferences and workshops including the International Conference on Parallel Processing, Reconfigurable Architecture Workshop, and Workshop on Advances in Parallel and Distributed Computational Models. His research interests includes parallel algorithms and architectures, hardware algorithms, mobile computing, computational complexity, and graph theory. He is a member of the IEEE Computer Society.

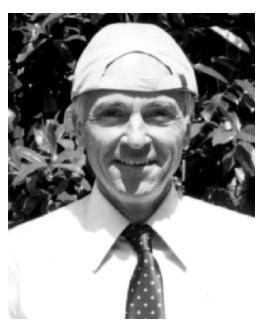

Stephan Olariu received the MSc and PhD degrees in computer science from McGill University, Montreal, in 1983 and 1986, respectively. In 1986, he joined the Computer Science Department at Old Dominion University, where he is now a professor. He has published extensively in various journals, book chapters, and conference proceedings. His research interests include wireless networks and mobile computing, parallel and distributed systems, performance evaluation, and medical image processing. He serves on the editorial boards of several archival journals including, the IEEE Transactions on Parallel and Distributed Systems, Journal of Parallel and Distributed Computing, International Journal of Foundations of Computer Science, Journal of Supercomputing, International Journal of Computer Mathematics, VLSI Design, and Parallel Algorithms and Applications. He is a member of the IEEE Computer Society. 


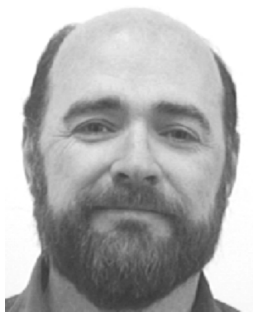

James L. Schwing (M '83) received the BS degree in mathematics from Worcester Polytechnic Institute in 1970 and the $\mathrm{PhD}$ degree in mathematics from the University of Utah in 1976. He is currently the chair of the Computer Science Department at Central Washington University. He serves on the editorial board of the International Journal of Computer Mathematics. His research interests include parallel algorithms, computer graphics, human-computer interaction, computer-aided design, and geo-spatial data conflation issues. He is a member of the IEEE Computer Society and the ACM.

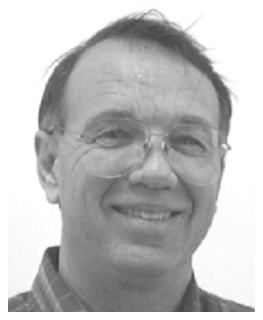

Larry Wilson received the BS degree from Midwestern State University in 1963 the MS and $\mathrm{PhD}$ degrees from the University of Texas at Austin in 1968 and 1971, respectively. He joined the Mathematics Department at Old Dominion University in 1968 and transferred to the Computer Science Department in 1979, where he is now an associate professor. He has published in the areas of Mikusinski operator functions, software reliability, parallel processing, and mobile computing. He is a member of the IEEE Computer Society. $\triangleright$ For further information on this or any computing topic, please
visit our Digital Library at http://computer.org/publications/dlib. 